\title{
Erythema nodosum as a presentation of inflammatory bowel disease
}

THE CASE: An 11-year-old girl had a 3-week history of painful, red nodules on her lower legs and a painful, swollen right foot that precluded weight-bearing. Fever developed on the day of presentation. She was previously well without a history of fatigue, weight loss, anorexia or night sweats. There was no history of trauma, medication use, known exposure to tuberculosis, upper respiratory tract infection, abdominal pain, bloody stools or diarrhea.

Physical examination revealed a thin but healthy looking prepubescent girl. Her weight was $26 \mathrm{~kg}$ (below the third percentile) and height $142 \mathrm{~cm}$ (10th to 25th percentile), and her growth curves demonstrated only a slight reduction in weight velocity. The skin lesions were on the anterior and posterior portions of both of the girl's lower legs (Fig. 1, arrows). There was substantial soft-tissue swelling, warmth and effusion in her right ankle. The patient had painful mouth ulcers, but her eyes, other joints and the rest of her skin were normal. Her abdominal and perianal examinations were unremarkable. Her hemoglobin level was 108 (normal 120-160) g/L, leukocyte count 8.6 (normal $4-10) \times 10^{9} / \mathrm{L}$, with a left shift (3.28 polymorphs, 1.18 bands), and mild thrombocytosis (platelet count 516 [normal 150-450] $\times 10^{\circ} / \mathrm{L}$ ). Her erythrocyte sedimentation rate was elevated at 112 (normal $1-10) \mathrm{mm} / \mathrm{h}$. A punch biopsy of a skin lesion revealed findings consistent with erythema nodosum. A subsequent endoscopy of the lower gastrointestinal tract revealed severe inflammation, ulceration and pseudopolyps of the ascending colon and terminal ileum (Fig. 2) and pathologic changes consistent with Crohn's disease. Prednisone therapy $(1 \mathrm{mg} / \mathrm{kg}$ daily) resulted in rapid resolution of the skin lesions and musculoskeletal symptoms. Six weeks after presentation the patient was asymptomatic and had gained $3 \mathrm{~kg}$; tapering of the prednisone therapy was initiated, with no flare-up of symptoms to date.
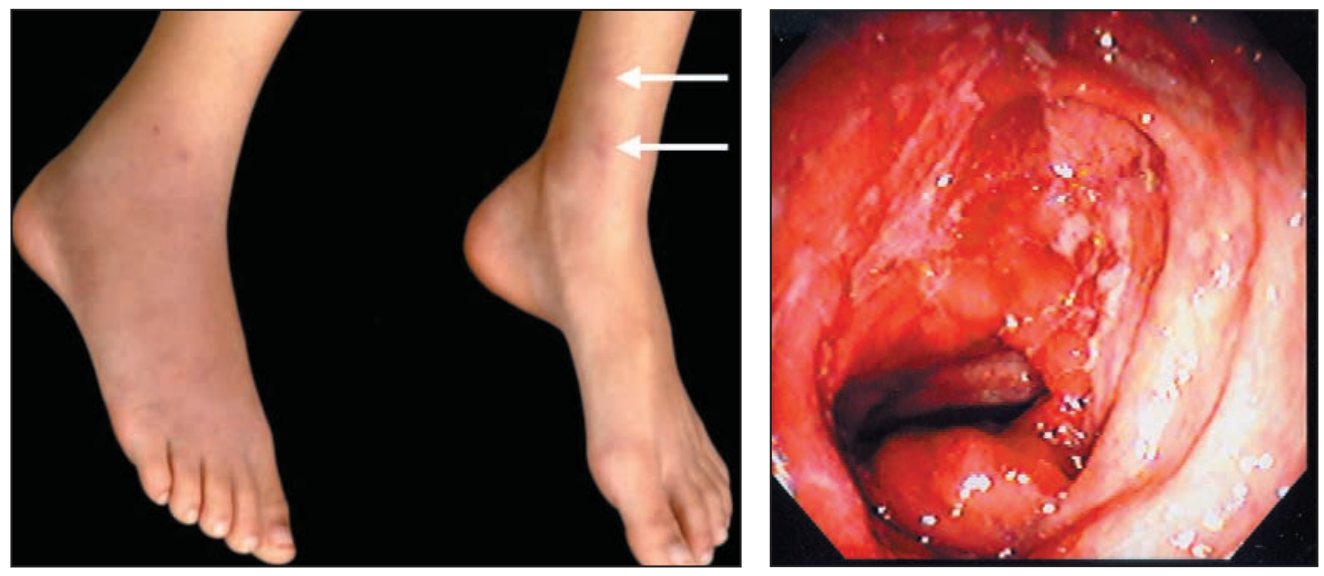

$\mathrm{T}$

his case was notable because the initial presentation of Crohn's disease consisted only of extraintestinal manifestations. This is uncommon, and the vast majority of children will present with the typical combination of abdominal pain, diarrhea and weight loss with or without extraintestinal manifestations (Box 1). Extraintestinal manifestations occur in about $25 \%-35 \%$ of cases of inflammatory bowel disease and may antedate any bowel symptoms, and they typically in- volve the musculoskeletal system (causing peripheral arthritis and spondyloarthropathy), skin, eyes (iritis and episcleritis) and hepatobiliary system. ${ }^{1}$ Arthritis and eye symptoms tend to correlate with intestinal inflammation and to resolve with treatment of the underlying bowel disease, whereas hepatobiliary disease correlates less well with intestinal disease activity. Erythema nodosum is the most common skin manifestation, occurring in about $10 \%-15 \%$ of people with inflammatory bowel disease, particularly those with Crohn's disease.

Erythema nodosum is an inflammatory process involving the subcutaneous fat lobules that causes tender, erythematous nodules that are usually seen symmetrically on the extensor surfaces of the lower extremities. Biopsy of a lesion typically shows fat necrosis or panniculitis (inflammation of the superficial fascia causing subcutaneous induration). Erythema nodosum is the most common form of panniculitis. It 
is an immunologic response to a variety of different antigens, with the underlying causes dependent on the population and geographic location.
Among children in developed countries, infections (predominantly streptococcal and EpsteinBarr virus infections) and inflammatory bowel disease are the

\section{Box 1: Modes of presentation of Crohn's disease in children (frequency)*}

- Classic presentation (abdominal pain, diarrhea, weight loss \pm extraintestinal manifestations) $(78.6 \%)$

- Extraintestinal manifestations predominating (8.4\%)

- Arthritis

- Recurrent fevers

- Recurrent oral ulcers

- Oral cheilitis

- Pyoderma gangrenosum

- Recurrent acute pancreatitis

- Perianal disease predominating (3.7\%)

- Growth failure predominating (3.3\%)

- Anemia predominating $(2.7 \%)$

- Anorexia, weight loss predominating $(2.0 \%)$

- Laparotomy for acute abdominal pain (1.3\%)

*Percentages are derived from data collected from 299 children presenting with Crohn's disease between 1980 and 1989 at the Hospital for Sick Children, Toronto. Adapted from Griffiths AM. Crohn's disease. In: David TJ, editor. Recent advances in pediatrics. Edinburgh: Churchill Livingston; 1992; p. 145.

\section{Box 2: Causes of erythema nodosum}

- Infection

- Bacterial: Group A streptococcus, cat scratch disease, tuberculosis, Yersinia, Salmonella, Shigella, Campylobacter, mycoplasma

- Viral: HIV, herpes simplex, hepatitis B and C, EpsteinBarr

- Fungal: histoplasmosis, coccidioidomycosis

- Protozoal: toxoplasmosis, amebiasis, giardiasis, ascariasis

- Rheumatologic disease: ulcerative colitis, Crohn's disease, Behçet's syndrome, sarcoidosis, spondyloarthropathy

- Malignant disease: lymphoma, leukemia

- Adverse drug reactions: sulfonamides, phenytoin, oral contraceptives, minocyclin, salicylates

- Idiopathic

Adapted from Camilleri MJ, Su WPD. Panniculitis. In: Freedberg IM, Eisen AZ, Wolff K, Austen KF, Goldsmith LA, Katz SI, editors. Fitzpatrick's dermatology in general medicine. New York: McGraw-Hill; 2003, p. 1055, and from Requena L, Requena C. Erythema nodosum. Dermatol Online J 2002;8(1):4.

most common causes of erythema nodosum. ${ }^{2}$ Tuberculosis has been found to be the most common association in areas where the disease is endemic. ${ }^{3}$ Other causes include reactions to drugs (e.g., sulfonamides), sarcoidosis, connective tissue disorders and malignant diseases (e.g., lymphoma). In up to $60 \%$ of cases, no underlying cause is found (Box 2).

The differential diagnosis of erythema nodosum includes erythema induratum, which typically affects adolescent and postmenopausal women and often produces ulcerative lesions on the calves; factitial panniculitis caused by self-inflicted trauma or injections; and lesions associated with pancreatic disease, $\alpha_{1}$-antitrypsin deficiency and lupus. Pyoderma gangrenosum, another dermatologic lesion associated with inflammatory bowel disease, differs from erythema nodosum because of its characteristic painful pustules and ulcers. Erythema nodosum generally resolves with treatment of the underlying condition or can resolve spontaneously in idiopathic cases.

\section{Michael Weinstein}

Department of Pediatrics

\section{Dan Turner}

Yaron Avitzur

Division of Gastroenterology, Hepatology and Nutrition Hospital for Sick Children Toronto, Ont.

\section{References}

1. Su CG, Judge TA, Lichtenstein GR Extraintestinal manifestations of inflammatory bowel disease. Gastroenterol Clin North Am 2002;31:307-27.

2. Garty BZ, Poznanski O. Erythema nodosum in Israeli children. Isr Med Assoc 7 2000;2:145-6.

3. Fox MD, Schwartz RA. Erythema nodosum. Am Fam Physician 1992;46: 818-22. 